\title{
ON A FAMILIAR THEOREM OF THE THEORY OF FUNCTIONS.
}

\author{
BY PROFESSOR EDMUND LANDAU.
}

In the second part of a paper* published in 1896, Professor Osgood considers the theorem :

"Let a function $f(z)$ of the complex variable $z$ be singlevalued and analytic at all points of the neighborhood of the point $z=c$ (exclusive of $z=c$ ), that is, for $0<|z-c|<\gamma$; also, for these values of $z$, let $|f(z)|$ remain less than some fixed value. Then $\lim _{z=c} f(z)=\alpha$ exists, and if at $z=c$ we assign to the function the value $\alpha$, then $f(z)$ will be analy tic at the point $c$. "

This follows easily, for example, from Laurent's theorem. Professor Osgood mentions the various proofs to be found in the literature of the subject and states that in several treatises the following fallacious proof is contained :

Let a function $\phi(z)$ be defined by the equations

$$
\phi(z)=(z-c) f(z)(0<|z-c|<\gamma), \quad \phi(c)=0 ;
$$

then by hypothesis, $\lim _{z=c} \phi(z)=0$, and $\phi(z)$ is continuous at the point $c$ and analytic for $0<|z-c|<\gamma$. From this some writers incorrectly infer that $\phi(z)$ is also analytic at $z=c$.

This conclusion is not justified, even when the existence of $\operatorname{iim}_{z=c} f(z)=\alpha$, or of

$$
\phi^{\prime}(c)=\lim _{z=c} \frac{(z-c) . f(z)}{z-c}=\alpha
$$

is presupposed. $\dagger$ For from the existence of the derivative of $\phi(z)$, without knowledge of its continuity, it could not be inferred, previously to 1900 , that $\phi(z)$ is analytic. Not until Goursat's \$ proof of Cauchy's integral theorem had appeared

* "Some points in the elements of the theory of functions." Bulletin, ser. 2 , vol. 2 , pp. 296-302.

+ This assumption is no restriction of the generality. For the function $(z-c) f(z)$ has a limiting value, namely zero, for $z=c$, and it is sufficient to show that this function is analytic at $z=c$.

‡ "Sur la définition générale des fonctions analytiques d'après Cauchy." Trans. Amer Math. Society, vol. 1 (1900), pp. 14-16. Compare the niore correct presentation of Goursat's proof by Pringsheim. "Ueber den Goursatschen Beweis des Cauchyschen Integralsatzes," ibid, vol. 2 (1901), pp. 413-421. 
was this conclusion, to which Professor Osgood quite rightly took exception in 1896, later justified.

At the end of his paper, Professor Osgood has added to the valid proofs of the theorem two new demonstrations.

I should like to communicate another proof, which stands in closer relation to the fallacious process of thought previously referred to, and which permits the gap to be filled without the use of Goursat's theorem.

There was lacking only the proof that the derivative $\phi^{\prime}(z)$ is continuous at the point $z=c$. Since for $0<|z-c|<\gamma$ we have

$$
\phi^{\prime}(z)=(z-c) f^{\prime}(z)+f(z)
$$

and since the existence of

$$
\lim _{z=c} f(z)=\alpha
$$

might be assumed, it only remains to show that

$$
\lim _{z=c}(z-c) f^{\prime}(z)
$$

exists and is equal to zero.

From (3), there exists for any previously chosen $\delta$ an $\epsilon$ such that for $0<|z-c|<\epsilon$ we have $|f(z)-\alpha|<\delta$. If we take $0<|z-c|<\frac{2}{3} \epsilon$, and apply Cauchy's formula to the function $f^{\prime}(z)-\alpha$, we get

$$
f^{\prime}(z)=\frac{1}{2 \pi i} \int \frac{f(x)-\alpha}{(x-z)^{2}} d x,
$$

where the integral is extended over the circumference with center at $z$ and radius $\mid z-c i / 2$. Hence follows

or

$$
\begin{gathered}
\left|f^{\prime}(z)\right|<\frac{1}{2 \pi} \cdot 2 \pi \frac{|z-c|}{2} \cdot \frac{\delta}{\frac{|z-c|^{2}}{4}}=\frac{2 \delta}{|z-c|}, \\
\left|(z-c) f^{\prime}(z)\right|<2 \delta
\end{gathered}
$$

$$
\lim _{z=c}(z-c) f^{\prime}(z)=0
$$

and, by use of (1), (2) and (3),

BERLIN,

$$
\lim _{z=c} \phi^{\prime}(z)=\alpha=\phi^{\prime}(c) .
$$

August 9, 1905. 